\title{
THE EFFECT OF NON-SURGICAL PERIODONTAL THERAPY ON SERUM RESISTIN LEVEL IN CHRONIC PERIODONTITIS PATIENTS WITH OR WITHOUT TYPE 2 DIABETES MELLITUS
}

\author{
Weam Elbattawy*, Sahar Mahmoud Abdel Raouf** and Shaimaa Saieed Nasr ${ }^{* * *}$
}

\begin{abstract}
Background: Resistin is a recognized marker for chronic inflammatory conditions that could be directly linked with type 2 diabetes mellitus. This study aimed to assess serum Resistin level in type 2 diabetic patients with chronic periodontitis after non-surgical periodontal therapy.

Methods: Forty individuals were recruited into three groups, group I consisted of 15 diabetic patients (type- 2 diabetes mellitus) with moderate to severe chronic periodontitis, and 15 non-diabetic patients with moderate to severe chronic periodontitis in group II and group III consisted of 10 nondiabetic individuals with healthy periodontium. Non-surgical periodontal therapy with supra and subgingival debridement was performed for groups I and II. Gingival index, plaque index, probing pocket depth, clinical attachment loss, serum Resistin and high sensitivity C-Reactive Protein assay were recorded for groups I and II before and after treatment, while HbA1c was recorded for group I.

Results: Both groups showed statistically significant improvement in all clinical periodontal parameters from baseline to 3 months with no statistically significant difference when comparing both groups throughout the clinical study. A highly statistically significant decrease in mean serum levels of Resistin and CRP after treatment in each group. Additionally, a statistically significant decrease in mean HbA1c level in group I was observed after treatment.
\end{abstract}

Conclusion: Non-surgical periodontal therapy was effective in improving clinical periodontal parameters, serum Resistin and C-reactive protein levels after 3 months for both diabetic and nondiabetic groups.

KEYWORDS: Chronic periodontitis, serum resistin, C-reactive protein, type 2 diabetes mellitus.

\footnotetext{
* M.Sc, Phd, Lecturer, Department of Oral Medicine and Periodontology, Faculty of Dentistry, Cairo University, Cairo, Egypt.

** M.Sc, Phd, Lecturer, Department of Oral Medicine, Faculty of Dentistry, Misr International University.

*** M.Sc, Phd, Lecturer, Department of Oral Medicine and Periodontology, Faculty of Dentistry, Fayoum University.
} 


\section{INTRODUCTION}

Chronic periodontitis results from the accumulation of periodontal pathogenic bacteria in dental plaque and its products that leads to local tissue destruction and host immunoinflammatory response. During this inflammatory process, there is infiltration of inflammatory cells which lead to production of certain proinflammatory cytokines like prostaglandin E2, interleukin-1, interleukin-6, tumor necrosis factor and Resistin; which would end up with clinical attachment loss (Buduneli et al., 2007).

Resistin, a cysteine rich protein present in the inflammatory zone, is an adipocytokine that was thought to be mainly produced by the adipocytes (Pischon et al., 2007). However, it was later found to be mainly expressed from polymorphonuclear leukocytes, macrophages and neutrophils; after being stimulated by lipopolysaccharides of periodontal pathogens (Filkova et al., 2009). Resistin level in serum increases with periodontal disease, subsequently, Resistin was found to augment the inflammatory process in periodontitis in the same way through pro-inflammatory agents, as it acts as a proinflammatory molecule by secreting interleukin-6, interleukin -12 and tumor necrosis factor- $\alpha$ (Gokhale et al., 2014).

Additionally, Resistin could exert distant systemic disorders such as increased insulin resistance which increases the risk for diabetes mellitus and hence the name Resistin was derived from observing its ability to induce insulin resistance in mice. On the other hand, chronic periodontitis is considered the sixth diabetic complication. Therefore, a bidirectional relationship between Resistin and periodontal disease existed (Saito et al., 2008).

In a study by (Adeghate, 2004) it was found that reducing Resistin can completely reverse the hepatic insulin resistance in diet-induced insulin resistant mice. In addition to another study by (Satoh et al., 2004), which concluded that overexpression of Resistin in circulation led to glucose intolerance, hyperinsulinemia, and hypertriglyceridemia. Therefore, chronic exposure to Resistin could affect the entry of glucose into the cells and counteract the effect of insulin.

(Devanoorkar et al., 2014) investigated the level of serum Resistin and found that its level in patients with chronic periodontitis was higher than healthy subjects. Additionally, in another conducted study, Resistin level in gingival crevicular fluid was higher in chronic periodontitis patients than in control group (Hiroshima et al., 2012).

C-reactive protein (CRP) has been considered in the research as an inflammatory mediator that may modify the inflammation in the acute phase response (Pasceri, Willerson and Yeh, 2000). This plasma protein has important biological functions, which are likely to be beneficial for survival and hence was used as a marker for identifying the ongoing inflammation (Pepys et al., 2006).

Moreover, it was evident that patients with chronic periodontitis showed high serum level of CRP; which was also found to impair the insulin signaling (Devaraj, Singh and Jialal, 2009). Subsequently, it may be involved in insulin resistance that may increase the risk of type 2 diabetes mellitus directly or indirectly via pro-inflammatory cytokines (Freeman et al., 2002) (Nakajima et al., 2010).

Therefore, it may be hypothesized that serum Resistin and CRP levels in patients with chronic periodontitis and ongoing systemic inflammatory disease as type 2 diabetes mellitus could be elevated as compared to periodontally healthy patients. Accordingly, our current study was conducted to assess the impact of non-surgical periodontal therapy on serum Resistin level in diabetic patients with chronic periodontitis versus patients with only chronic periodontitis; and to emphasize their effect on glycemic control in type 2 diabetes mellitus. 


\section{MATERIALS AND METHODS}

\section{Study Design}

This clinical study was designed for comparing clinical and serological values of non-surgical periodontal therapy for management of moderate to severe chronic periodontitis in diabetic and nondiabetic patients. The study protocol was registered in ClinicalTrials.gov (ID: NCT04473209) and approved by the Research Ethics Committee of Faculty of Dentistry, Cairo University (Number: 6-5-20)

\section{Study Population}

The present clinical trial included 40 individuals ranging in age from 40 to 55 years old. Recruitment of subjects was performed from the outpatient clinic, Department of Oral Medicine and Periodontology, Faculty of Dentistry, Cairo University between November 2018 and September 2019. Three groups were included in this study; where 15 patients suffering from both type 2 diabetes mellitus and chronic periodontitis were categorized as group I, group II consisted of 15 non-diabetic patients suffering from chronic periodontitis and group III consisted of 10 non-diabetic individuals with healthy periodontium.

Subjects who participated in this study except for group III met the following eligibility criteria: 1) diagnosed with moderate to severe chronic periodontitis (Armitage, 1999). 2) Presence of a minimum of 15 natural teeth, 3) Presence of at least four teeth with one or more sites with probing pocket depth (PPD) $\geq 5 \mathrm{~mm}$ and clinical attachment level (CAL) $\geq 4 \mathrm{~mm}$ and 3 ) agreed to sign a written informed consent. Regarding the exclusion criteria; 1) Patients who received any periodontal treatment or professional scaling and root planning of the teeth for at least 6 months prior to the study initiation (Devanoorkar et al., 2012); 2) A history of antibiotics therapy and anti-inflammatory drugs within the previous 6 months; 3) Pregnancy or use of contraceptives or any medication related to diabetes; and 4) former or current smokers $<5$ years.

\section{Pretreatment phase}

In the first visit, a detailed case history and full mouth initial examination was performed for the recruited participants to confirm the diagnosis of moderate to severe chronic periodontitis. Fasting blood glucose (FBG) level was recorded from diabetic patients (group I) to confirm diagnosis of type 2 diabetes mellitus according to the American Diabetes Association (ADA) ('Diagnosis and classification of diabetes mellitus,' 2004). Tooth brushing twice a day with a toothbrush and interdental floss were prescribed as oral hygiene instructions.

\section{Blinding}

The blinding process was implemented through the outcome assessor and the statistician who were blinded to the allocation and grouping of patients.

\section{Collection of blood samples}

Blood samples were collected from all patients into serum separator tubes. These samples were transferred immediately to the laboratory at baseline and 3 months post therapy.

\section{Treatment phase with non-surgical periodontal therapy (for groups I and II):}

Ultrasonic device with supragingival scaling inserts ${ }^{*}$ were used for full mouth debridement supra and subgingivally followed by subgingival debridement with periodontal area-specific curettes** Periodontal therapy was performed over two weeks. Instructions were given for proper plaque control

\footnotetext{
* EMS Woodpecker ultrasonic scaler tip, Woodpecker, China.

** HuFriedy Gracy's and mini curettes; HuFriedy, Chicago, USA.
} 
with tooth brushing and interdental cleaning aids. Chlorhexidine gluconate $(0.12 \%)^{*}$ was prescribed as a mouth rinse for patients to be used twice daily for two weeks. During the experimental period, patients in group I were instructed not to change the medications related to diabetes mellitus therapy and alteration of lifestyle, including exercise and diet should be known.

\section{Primary outcomes}

Serum Resistin level.

\section{Secondary outcomes:}

Clinical periodontal parameters plaque index $(\mathrm{PI})$, gingival index (GI), probing pocket depth (PD), clinical attachment loss (CAL), high sensitivity serum C-Reactive Protein Assay (CRP) and HbA1c.

\section{Clinical periodontal parameters}

All clinical parameters were measured at baseline and 3 months postoperatively by a single examiner (RM) who was blinded. The periodontal parameters plaque index (PI), gingival index (GI), PD and CAL were recorded for all participants according to (Silness and Loe, 1964). PD and CAL were measured using William's graduated periodontal probe and were approximated to the highest whole millimeter. PD was measured from free-gingival margin to base of the pocket and CAL was measured from cemento-enamel junction (CEJ) to base of the pocket. Measurements were recorded at six sites for all teeth mesio-buccal, mesio-lingual, mid-buccal, disto-buccal, disto-lingual, and mid-lingual.

Calculation of the PD score of an individual tooth was obtained by totaling of the PD measured per tooth surfaces and dividing by the number of surfaces examined. Then the same procedure was performed to calculate the CAL score for each tooth.
For the PI score, the score of a tooth was obtained by totaling of plaque score per unit and dividing by four. Score of an individual was obtained by totaling of the plaque score per tooth and dividing by the number of teeth examined. And the same procedure was performed to calculate the GI score.

\section{Assay procedure}

Measurement of Glycated hemoglobin (Hb Alc):

Patients from group I were assessed for glycated hemoglobin (HbA1c) at baseline and 3 months postoperatively.

\section{Measurement of high sensitivity serum C-Reactive Protein Assay (CRP):}

Microtiter strips coated with anti-CRP antibody were incubated with diluted standard sera and patient samples. During this incubation step, CRP was bound specifically to the wells. After removal of the unbound serum proteins by a washing procedure, the antigen-antibody complex in each well was detected with specific peroxidase-conjugated antibodies after removal of the unbound conjugate, the strips were incubated with a chromogen solution containing tetramethylbenzidine and hydrogen peroxide. A blue color developed in proportion to the amount of immunocomplex bound to the wells of the strips. The enzymatic reaction was stopped by the addition of $\left(2 \mathrm{~N} \mathrm{H}_{2} \mathrm{So}_{4}\right)$ and the absorbance values at $450 \mathrm{~nm}$ were determined. A standard curve was obtained by plotting the absorbance values versus the corresponding standard values. The concentration of CRP in patient samples was determined by interpolation from the standard curve.

\section{Measurement of Serum Resistin levels}

Serum Resistin was measured using a human Resistin enzyme-linked immunosorbent assay

\footnotetext{
* Hexitol; Chlorhexidine HCL mouthwash, The Arab Drug Company for pharmaceutical \& CHEM. IND. CO. CairoEgypt.
} 
kit (BioVendor research and diagnostic product) following the manufacturer's protocol. In the BioVendor Human Resistin ELISA, standards and samples are incubated in microplate wells pre-coated with polyclonal anti-human Resistin antibody. After one-hour incubation and washing, biotin labelled second polyclonal anti-human Resistin antibody is added and incubated with captured Resistin for one hour. After another washing, streptavidin-HRP conjugate is added. After one-hour incubation and the last washing step, the remaining conjugate is allowed to react with the substrate solution (TMB). The reaction is stopped by addition of acidic solution and absorbance of the resulting yellow product is measured. The absorbance is proportional to the concentration of Resistin. A standard curve is constructed by plotting absorbance values against concentrations of standards, and concentrations of unknown samples are determined using this standard curve.

\section{Reevaluation}

After 3 months of non- surgical periodontal therapy, groups I and II were recalled for reevaluation of clinical parameters, serum Resistin, and CRP (Rode et al., 2019). In addition to HbA1c was evaluated in group I (diabetic group) after the same follow up period.

\section{Sample size calculation}

The present study aimed at assessing how nonsurgical periodontal therapy could affect the level of serum Resistin in chronic periodontitis patients with diabetes mellitus type 2 versus patients with only chronic periodontitis. Sample size was calculated according (Gokhale et al., 2014) and using G-power program, the effect size between both groups was found to be 1.3 using power of $80 \%$ and $5 \%$ significance level giving a total sample size of 22 patients (11 patients in each group). This number was to be increased to a total sample size of 30 (15 patients per group) to compensate for possible losses or drop out during the follow up.

\section{Statistical and power analysis}

Values of clinical parameters were presented in the form of mean and standard deviation (SD). Probing pocket depth (PD), clinical attachment loss (CAL) and HbA1c data showed normal (parametric) distribution, so the two groups were compared using Student's t-test. while, the changes by time within each group were studied by the Paired t-test.

Data of plaque Index (PI), Gingival Index (GI), and percentage change showed non-parametric distribution, thus comparing between both groups was performed using the Mann-Whitney U test, which is considered a non-parametric alternative to Student's t-test. Changes by time within each group were studied using Wilcoxon signed-rank test, which is considered as a non-parametric alternative to paired t-test.

Regarding data of CRP and Resistin in all groups were subjected to statistical analyses of variance (F-test) "one-way ANOVA". It is a procedure that was used for testing the difference among the means of the three groups. Based on the P-value, we can figure out if the difference was significant or not.

Moreover, Duncan's multiple range test was used among groups, where means followed by same superscript letters, indicate that there was no significant difference between means. However, means with different superscript letters indicate presence of significant difference.

\section{RESULTS}

\section{Clinical periodontal parameters}

The changes in clinical periodontal parameters in both study groups are presented in table (1). The mean PD values in groups I and II were reduced postoperatively, with statistically significant difference among each group. When comparing the PD values between both groups after 3 months; a statistically significant difference was found with $P$ value 0.024 . 
Regarding the mean CAL values, they were reduced in the two groups postoperatively and a statistically significant difference was found. However, more reduction in the mean CAL values was revealed in group II than in group I and a statistically significant difference was found with Pvalue $=0.01$ on comparing the two groups .

Regarding PI and GI as shown in table (1), there was an obvious decrease in mean scores after treatment among each group, with a statistically significant difference in PI $(P$-value $=0.008)$ for group I, $(P$-value $=0.012)$ for group II; while P-value for GI was 0.008 in group I and 0.012 in group II. No statistically significant difference was found between the mean scores of groups I and II when comparing them pre- and postoperatively, with P-value 0.773 and 0.847 respectively for PI; and P-value 0.815 and 0.541 respectively for GI.

\section{Glycated hemoglobin (Hb A1c):}

Table (2) shows the mean and SD values of $\mathrm{HbA1c}$ in diabetic patients with periodontitis (group I). The preoperative mean value was $(7.2 \pm 0.189)$ while postoperatively the mean value of $\mathrm{HbA} 1 \mathrm{c}$ was (3.507 \pm 0.111$)$, which showed a statistically significant decrease in mean $\mathrm{HbA} 1 \mathrm{c}$ after treatment in Group I.

\section{High sensitivity serum C-Reactive Protein Assay (CRP):}

In table (3), it was shown that mean reduction in serum CRP after treatment in both groups I \& II was statistically significant among each group at P- value 0.000. However, when comparing the mean reduction values of CRP of the two groups, no statistically significant difference was found. When comparing the two groups with the control one postoperatively, there was a statistically significant difference at P-value 0.001 after 3 months; as the control group has the lowest mean value.

TABLE (1): Mean, standard deviation and percentage reduction of clinical periodontal outcomes (PD, CAL, PI and GI) in both studied groups throughout the experimental period:

\begin{tabular}{|c|c|c|c|c|}
\hline & Baseline & 3 months & P-value & percentage reduction $\%$ \\
\hline \multicolumn{5}{|c|}{$\mathrm{PD}($ mean $\pm \mathrm{SD})$} \\
\hline Group I & $3.5 \pm 0.6$ & $2.8 \pm 0.8$ & $0.001 *$ & $20.3 \pm 12.3$ \\
\hline Group II & $2.6 \pm 0.5$ & $1.9 \pm 0.6$ & $0.001 *$ & $29.4 \pm 14.1$ \\
\hline P-value & $0.008 *$ & $0.024 *$ & & 0.236 \\
\hline \multicolumn{5}{|c|}{ CAL $($ mean \pm SD) } \\
\hline Group I & $5 \pm 1.3$ & $4.1 \pm 1.1$ & $0.001 *$ & $16.9 \pm 9.5$ \\
\hline Group II & $3.4 \pm 0.6$ & $2.7 \pm 0.8$ & $0.011 *$ & $20.6 \pm 16.6$ \\
\hline P-value & $0.006 *$ & $0.010 *$ & & 0.606 \\
\hline \multicolumn{5}{|c|}{ PI $($ mean \pm SD $)$} \\
\hline Group I & $2.11 \pm 0.4$ & $1.3 \pm 0.3$ & $0.008 *$ & $36.7 \pm 19.1$ \\
\hline Group II & $2.06 \pm 0.2$ & $1.35 \pm 0.4$ & $0.012 *$ & $34.9 \pm 15.3$ \\
\hline P-value & 0.773 & 0.847 & & 0.963 \\
\hline \multicolumn{5}{|c|}{ GI (mean \pm SD) } \\
\hline Group I & $2.66 \pm 0.5$ & $1.68 \pm 0.5$ & $0.008 *$ & $35 \pm 20.6$ \\
\hline Group II & $2.74 \pm 0.5$ & $1.98 \pm 0.6$ & $0.012 *$ & $29.2 \pm 12.3$ \\
\hline P-value & 0.815 & 0.541 & & 0.541 \\
\hline
\end{tabular}

*: Significant at $P \leq 0.05$ 
TABLE (2): Mean and standard deviation for the changes in $\mathrm{HbA1c}$ after treatment in group I:

\begin{tabular}{ccccc}
\hline & Baseline & 3 months & P-value & T-value \\
\hline HbA1c & & & & \\
$(\mathbf{4 . 2 - 6 . 2 \% )}$ & & & & \\
$($ mean \pm SD $)$ & & & & \\
Group I & $7.2 \pm 0.189$ & $3.507 \pm 0.111$ & 0.000 & 20.132 \\
\hline
\end{tabular}

Statistically highly significant difference at $P<0.001$

\section{Serum Resistin level}

Table (3) shows the changes in serum Resistin levels throughout the study period. At baseline, group I (diabetic group) showed the highest mean values for serum Resistin $\left(16.846^{\mathrm{a}} \pm 0.566\right)$, while group II showed statistically significant lower values $\left(12.118^{\mathrm{a}} \pm 0.202\right)$ at baseline, while the control healthy group showed the least serum Resistin level $\left(6.916^{\mathrm{c}} \pm 0.181\right)$. After non-surgical periodontal treatment, an obvious statistically significant decrease in serum Resistin level in group I $(P=0.000)$ and group II $(P=0.000)$ was found, but with no statistically significant difference between the two groups postoperatively. On comparing the three studied groups at different time intervals, a statistically significant difference between them was observed $(P=0.000)$.

\section{DISCUSSION}

Chronic periodontitis can aggravate the glycemic control in type 2 diabetic patients; through the release of various inflammatory mediators such as serum Resistin and CRP that are responsible for increasing insulin resistance (Bharti et al., 2013). Also, it is well established that chronic exposure to Resistin could adversely affect the glucose entry into cells, thus counteracting the effect of insulin (Sanchez-Solana, Laborda and Baladron, 2012). Therefore, the levels of Resistin and CRP were observed in this clinical trial after non-surgical periodontal therapy. Chronic periodontitis patients with diabetes mellitus type 2 versus non-diabetic ones were included to assess the glycemic control as well.

Results of the current study showed a significant improvement after non-surgical periodontal therapy in PD, CAL, PI, and GI in groups I and II. This

TABLE (3): Changes in serum Resistin and CRP levels in all studied groups throughout the study period:

\begin{tabular}{lcccc}
\hline & Baseline & 3 months & P-value & F-value \\
\hline CRP $($ ug $/$ ml) $($ mean \pm SD) & & & & \\
Group I & $12.241^{\mathrm{a}} \pm 0.803$ & $8.475^{\mathrm{b}} \pm 0.893$ & 0.000 & 39.382 \\
Group II & $6.374^{\mathrm{a}} \pm 0.359$ & $3.198^{\mathrm{b}} \pm 0.207$ & 0.000 & 76.266 \\
Control III (control) & $2.085^{\mathrm{c}} \pm 0.550$ & $2.085^{\mathrm{c}} \pm 0.550$ & & \\
P-value & 0.000 & 0.001 & & \\
F-value & 19.215 & 7.972 & & \\
\hline Resistin (ng/ml) (mean \pm SD) & & & & \\
Group I & $16.846^{\mathrm{a}} \pm 0.566$ & $9.353^{\mathrm{b}} \pm 0.460$ & 0.000 & 125.611 \\
Group II & $12.118^{\mathrm{a}} \pm 0.202$ & $7.287^{\mathrm{b}} \pm 0.163$ & 0.000 & 253.518 \\
Control III (control) & $6.916^{\mathrm{c}} \pm 0.181$ & $6.916^{\mathrm{c}} \pm 0.181$ & & \\
P-value & 0.000 & 0.000 & & \\
F-value & 161.48 & 17.82 & & \\
\hline
\end{tabular}

Statistically highly significant difference at $P<0.001$, Statistically significant difference at $P<0.05$, Different superscripts in the same column are statistically significantly different. 
improvement could be attributed to reduction of the inflammatory cytokines following intense periodontal debridement (Hammerle, Joss and Lang, 1991; Hughes and Caffesse, 1978; Cugini et al., 2000). However, PD and CAL in group II showed better improvement than in group I. This could be justified that diabetic patients in group I had more deterioration in the periodontal health than non-diabetic patients, due to release of more inflammatory cytokines. This was in accordance to Badersten et al., who concluded that diabetes mellitus had an impact on initiating the inflammatory process; leading to lesser improvement in clinical parameters after periodontal therapy when compared to non- diabetic subjects (Badersten, Nilveus and Egelberg, 1981).

Regarding serum Resistin and CRP levels in group I and II; they were observed at baseline then after 3 months post non-surgical periodontal therapy. Both markers were higher at baseline in groups I and II compared to the healthy group, this could be explained on the basis that Resistin and CRP are considered as potential markers for inflammation and affected by the inflammatory cytokines released from periodontal pathogens in chronic periodontitis. This was in agreement with (Tokuyama et al., 2007) and with a systematic review published in 2017 by (Akram et al., 2017), which concluded that Resistin levels in patients with chronic periodontitis and systemic inflammatory disorders such as diabetes, was high but not significantly higher than the levels in patients with only chronic periodontitis.

There was a significant reduction in serum Resistin and CRP levels post-therapy in both groups after 3 months. However, the mean reduction of these markers in diabetic group (group I) was lesser than those in group II. This could be due to the presence of a double way relationship between these markers and diabetes mellitus. Consequently, the inflammatory process could be accentuated in periodontitis patients with type 2 diabetes mellitus; that would in return influence Resistin and CRP expression. This on the other hand leads to insulin resistance and thus deterioration of glycemic control in diabetic patients would result (Devanoorkar et al., 2012).

Another study by (Rode et al., 2019) proved that in diabetic patients with chronic periodontitis, insulin resistance was enhanced by proinflammatory cytokines leading to increasing the inflammatory burden when compared to non-diabetic patients. Therefore, the mean reduction in serum Resistin and CRP levels after therapy were lower in chronic periodontitis patients with diabetes mellitus type 2 than in non-diabetic subjects with chronic periodontitis, however there was no statistically significant difference between the two groups.

Noteworthy, although there was improvement in serum Resistin and CPR levels after non- surgical periodontal therapy in both groups, they did not reach the normal level; when comparing the results with those of the control group (healthy subjects). This could be due to the presence of residual sites having high bacterial load enough to maintain the systemic inflammation even after therapy, and this was in agreement with a study by (Goncalves et al., 2015).

According to (Dag et al., 2009); since periodontal therapy appeared to reduce the periodontal infection and inflammation, therefore, it could reduce the levels of peripheral inflammatory cytokines and hence improve the insulin sensitivity. Thus, it would be suggested that periodontal therapy might be able to facilitate the metabolic control of diabetes mellitus type 2 as proven in several studies by (Sun et al., 2011; Lin et al., 2012; Moeintaghavi et al., 2012). Also, it was confirmed by (Pérez et al., 2017) that scaling and root planning could reduce periodontal pathogens and thus inhibit cytokines associated with inflammatory markers leading to decrease glucose level. However, this was not in agreement 
with (Chen et al., 2012), who could not find a strong association between the treatment of periodontal disease and the metabolic improvement in type 2 diabetes mellitus despite the large sample size and the long follow up period. Thus, it was clarified in the systematic review by (Mauri-Obradors et al., 2015) that other factors were able to modify the periodontal treatment outcomes such as; the type and duration of diabetes mellitus, initial glycemic status, type of treatment protocol, smoking and the initial periodontal status.

Accordingly, non -surgical periodontal therapy could be considered as a non-invasive approach that could improve the periodontal condition and reduce the level of inflammatory mediators. Also, periodontal therapy could have an impact on improving the inflammatory status systemically by reducing the serum Resistin and CRP. On the other hand, scaling and root planning could help in enhancing the glycemic control and was significantly better than no treatment in improving $\mathrm{HbA1c} \%$ in diabetic patients as confirmed in a recent systematic review and metanalysis by (Cao et al., 2019). However, this should be considered beside other factors, such as patient's compliance to strict oral hygiene measures and maintaining follow up visits.

\section{CONCLUSION}

Within the limitations of this study, it could be concluded that non-surgical periodontal therapy is effective in improving all the clinical periodontal parameters after 3 months for both diabetic and nondiabetic patients with moderate to severe chronic periodontitis as well as reducing $\mathrm{HbA} 1 \mathrm{c}$ in diabetic patients type 2. Serum Resistin and CRP could be significantly reduced after non-surgical periodontal therapy, however still they did not reach the normal levels as in healthy individuals.

\section{REFERENCES}

- Adeghate, E. (2004) 'An update on the biology and physiology of resistin', Cell Mol Life Sci, 61(19-20), pp. 2485-96.

- $\quad$ Akram, Z., Rahim, Z. H., Taiyeb-Ali, T. B., Shahdan, M. S., Baharuddin, N. A., Vaithilingam, R. D. and Safii, S. H. (2017) 'Resistin as potential biomarker for chronic periodontitis: A systematic review and meta-analysis', Arch Oral Biol, 73, pp. 311-320.

- Armitage, G. C. (1999) 'Development of a Classification System for Periodontal Diseases and Conditions', Annals of Periodontology, 4(1), pp. 1-6.

- $\quad$ Badersten, A., Nilveus, R. and Egelberg, J. (1981) 'Effect of nonsurgical periodontal therapy. I. Moderately advanced periodontitis', J Clin Periodontol, 8(1), pp. 57-72.

- $\quad$ Bharti, P., Katagiri, S., Nitta, H., Nagasawa, T., Kobayashi, H., Takeuchi, Y., Izumiyama, H., Uchimura, I., Inoue, S. and Izumi, Y. (2013) 'Periodontal treatment with topical antibiotics improves glycemic control in association with elevated serum adiponectin in patients with type 2 diabetes mellitus', Obes Res Clin Pract, 7(2), pp. e129-e138.

- Buduneli, E., Vardar-Sengul, S., Buduneli, N., Atilla, G., Wahlgren, J. and Sorsa, T. (2007) 'Matrix Metalloproteinases, Tissue Inhibitor of Matrix Metalloproteinase-1, and Laminin-5 gamma2 Chain Immunolocalization in Gingival Tissue of Endotoxin-Induced Periodontitis in Rats: Effects of Low-Dose Doxycycline and Alendronate', J Periodontol, 78(1), pp. 127-134.

- Cao, R., Li, Q., Wu, Q., Yao, M., Chen, Y. and Zhou, H. 'Effect of non-surgical periodontal therapy on glycemic control of type 2 diabetes mellitus: a systematic review and Bayesian network meta-analysis'. BMC Oral Health 19, 176 (2019). https://doi.org/10.1186/s12903019-0829-y

- Chen, L., Luo, G., Xuan, D., Wei, B., Liu, F., Li, J. and Zhang, J. (2012) 'Effects of non-surgical periodontal treatment on clinical response, serum inflammatory parameters, and metabolic control in patients with type 2 diabetes: a randomized study', J Periodontol, 83(4), pp. 435-43.

- $\quad$ Cugini, M. A., Haffajee, A. D., Smith, C., Kent, R. L., Jr. and Socransky, S. S. (2000) 'The effect of scaling and root planing on the clinical and microbiological parameters of periodontal diseases: 12-month results', J Clin Periodontol, 27(1), pp. 30-6. 
- $\quad$ Dag, A., Firat, E. T., Arikan, S., Kadiroglu, A. K. and Kaplan, A. (2009) 'The effect of periodontal therapy on serum TNF-alpha and HbA1c levels in type 2 diabetic patients', Aust Dent J, 54(1), pp. 17-22.

- Devanoorkar, A., Dwarakanath, C. D., Gundanavar, G., Kathariya, R. and Patil, S. R. (2012) 'Evaluation of serum resistin levels in periodontal health and disease and effects of non surgical periodontal therapy on its levels', Dis Markers, 32(5), pp. 289-94.

- Devanoorkar, A., Kathariya, R., Guttiganur, N., Gopalakrishnan, D. and Bagchi, P. (2014) 'Resistin: A Potential Biomarker for Periodontitis Influenced Diabetes Mellitus and Diabetes Induced Periodontitis', Disease Markers, 2014, pp. 7.

- Devaraj, S., Singh, U. and Jialal, I. (2009) 'Human Creactive protein and the metabolic syndrome', Curr Opin Lipidol, 20(3), pp. 182-9.

- 'Diagnosis and classification of diabetes mellitus', (2004) Diabetes Care, 27 Suppl 1, pp. S5-s10.

- $\quad$ Filkova, M., Haluzik, M., Gay, S. and Senolt, L. (2009) 'The role of resistin as a regulator of inflammation: Implications for various human pathologies', Clin Immunol, 133(2), pp. 157-70.

- $\quad$ Freeman, D. J., Norrie, J., Caslake, M. J., Gaw, A., Ford, I., Lowe, G. D., O’Reilly, D. S., Packard, C. J. and Sattar, N. (2002) 'C-reactive protein is an independent predictor of risk for the development of diabetes in the West of Scotland Coronary Prevention Study', Diabetes, 51(5), pp. 1596-600

- Gokhale, N. H., Acharya, A. B., Patil, V. S., Trivedi, D. J., Setty, S. and Thakur, S. L. (2014) 'Resistin levels in gingival crevicular fluid of patients with chronic periodontitis and type 2 diabetes mellitus', J Periodontol, 85(4), pp. 610-7.

- Goncalves, T. E., Zimmermann, G. S., Figueiredo, L. C., Souza Mde, C., da Cruz, D. F., Bastos, M. F., da Silva, H. D. and Duarte, P. M. (2015) 'Local and serum levels of adipokines in patients with obesity after periodontal therapy: one-year follow-up', J Clin Periodontol, 42(5), pp. 431-9.

- Hammerle, C. H., Joss, A. and Lang, N. P. (1991) 'Shortterm effects of initial periodontal therapy (hygienic phase)', J Clin Periodontol, 18(4), pp. 233-9.

- Hiroshima, Y., Bando, M., Inagaki, Y., Mihara, C., Kataoka, M., Murata, H., Shinohara, Y., Nagata, T. and Kido,
J. (2012) 'Resistin in gingival crevicular fluid and induction of resistin release by Porphyromonas gingivalis lipopolysaccharide in human neutrophils', J Periodontal Res, 47(5), pp. 554-62.

- $\quad$ Hughes, T. P. and Caffesse, R. G. (1978) 'Gingival changes following scaling, root planning and oral hygiene. A biometric evaluation', J Periodontol, 49(5), pp. 245-52.

- $\quad$ Lin, S. J., Tu, Y. K., Tsai, S. C., Lai, S. M. and Lu, H. K. (2012) 'Non-surgical periodontal therapy with and without subgingival minocycline administration in patients with poorly controlled type II diabetes: a randomized controlled clinical trial', Clin Oral Investig, 16(2), pp. 599-609.

- Mauri-Obradors, E., Jané-Salas, E., Sabater-Recolons Mdel, M., Vinas, M. and López-López, J. (2015) 'Effect of nonsurgical periodontal treatment on glycosylated hemoglobin in diabetic patients: a systematic review', Odontology, 103(3), pp. 301-13.

- Moeintaghavi, A., Arab, H. R., Bozorgnia, Y., Kianoush, K. and Alizadeh, M. (2012) 'Non-surgical periodontal therapy affects metabolic control in diabetics: a randomized controlled clinical trial', Aust Dent J, 57(1), pp. 31-7.

- Nakajima, T., Honda, T., Domon, H., Okui, T., Kajita, K., Ito, H., Takahashi, N., Maekawa, T., Tabeta, K. and Yamazaki, K. (2010) 'Periodontitis-associated up-regulation of systemic inflammatory mediator level may increase the risk of coronary heart disease', J Periodontal Res, 45(1), pp. 116-22.

- $\quad$ Pasceri, V., Willerson, J. T. and Yeh, E. T. (2000) 'Direct proinflammatory effect of C-reactive protein on human endothelial cells', Circulation, 102(18), pp. 2165-8.

- $\quad$ Pepys, M. B., Hirschfield, G. M., Tennent, G. A., Gallimore, J. R., Kahan, M. C., Bellotti, V., Hawkins, P. N., Myers, R. M., Smith, M. D., Polara, A., Cobb, A. J., Ley, S. V., Aquilina, J. A., Robinson, C. V., Sharif, I., Gray, G. A., Sabin, C. A., Jenvey, M. C., Kolstoe, S. E., Thompson, D. and Wood, S. P. (2006) 'Targeting C-reactive protein for the treatment of cardiovascular disease', Nature, 440(7088), pp. 1217-21.

- Pischon, N., Heng, N., Bernimoulin, J. P., Kleber, B. M., Willich, S. N. and Pischon, T. (2007) 'Obesity, inflammation, and periodontal disease', J Dent Res, 86(5), pp. 400-9.

- $\quad$ Pérez, C. M., Muñoz, F., Andriankaja, O. M., Ritchie, C. S., Martínez, S., Vergara, J., Vivaldi, J., López, L., Campos, M. and Joshipura, K. J. (2017) 'Cross-sectional associations of impaired glucose metabolism measures with 
bleeding on probing and periodontitis', J Clin Periodontol, 44(2), pp. 142-149.

- $\quad$ Rode, P. A., Kolte, R. A., Kolte, A. P., Purohit, H. J. and Ahuja, C. R. (2019) 'Relevance of single-nucleotide polymorphism to the expression of resistin gene affecting serum and gingival crevicular fluid resistin levels in chronic periodontitis and type 2 diabetes mellitus: A randomized control clinical trial', J Indian Soc Periodontol, 23(2), pp. 131-136.

- $\quad$ Saito, T., Yamaguchi, N., Shimazaki, Y., Hayashida, H., Yonemoto, K., Doi, Y., Kiyohara, Y., Iida, M. and Yamashita, Y. (2008) 'Serum levels of resistin and adiponectin in women with periodontitis: the Hisayama study', J Dent Res, 87(4), pp. 319-22.

- Sanchez-Solana, B., Laborda, J. and Baladron, V. (2012) 'Mouse resistin modulates adipogenesis and glucose uptake in 3T3-L1 preadipocytes through the ROR1 receptor', Mol Endocrinol, 26(1), pp. 110-27.
- $\quad$ Satoh, H., Nguyen, M. T. A., Miles, P. D. G., Imamura, T., Usui, I. and Olefsky, J. M. (2004) 'Adenovirus-mediated chronic "hyper-resistinemia" leads to in vivo insulin resistance in normal rats', The Journal of clinical investigation, 114(2), pp. 224-231.

- $\quad$ Silness, J. and Loe, H. (1964) 'PERIODONTAL DISEASE IN PREGNANCY. II. CORRELATION BETWEEN ORAL HYGIENE AND PERIODONTAL CONDTION', Acta Odontol Scand, 22, pp. 121-35.

- $\quad$ Sun, W. L., Chen, L. L., Zhang, S. Z., Wu, Y. M., Ren, Y.Z. and Qin, G. M. (2011) 'Inflammatory cytokines, adiponectin, insulin resistance and metabolic control after periodontal intervention in patients with type 2 diabetes and chronic periodontitis', Intern Med, 50(15), pp. 1569-74.

- $\quad$ Tokuyama, Y., Osawa, H., Ishizuka, T., Onuma, H., Matsui, K., Egashira, T., Makino, H. and Kanatsuka, A. (2007) 'Serum resistin level is associated with insulin sensitivity in Japanese patients with type 2 diabetes mellitus', Metabolism, 56(5), pp. 693-8. 